\title{
First results of X-shooter observations of IGR sources
}

\section{P. Goldoni ${ }^{* \dagger}$}

APC, Univ. Paris Diderot, CNRS/IN2P3, CEA/IRFU, Obs. de Paris, Sorbonne Paris Cité, France

E-mail: goldonidapc.univ-paris7.fr

\section{S. Chaty}

Laboratoire AIM (UMR-E 9005 CEA/DSM-CNRS-Université Paris Diderot), Irfu/Service d'Astrophysique, CEA-Saclay and Institut Universitaire de France, 103, bd Saint Michel, Paris, France

\section{A. Goldwurm}

APC, Univ. Paris Diderot, CNRS/IN2P3, CEA/IRFU, Obs. de Paris, Sorbonne Paris Cité, France

\section{A. Coleiro}

Laboratoire AIM (UMR-E 9005 CEA/DSM-CNRS-Université Paris Diderot), Irfu/Service d'Astrophysique, CEA-Saclay

\begin{abstract}
X-shooter is a second generation ESO-VLT instrument that had its first light in October 2009. It is a single object medium-resolution spectrograph whose main feature is the capability of covering simultaneously in a single observation the range from 3000 to 24000 Angstrom. This unique capability is very well suited to investigate the complex spectra of the optical counterparts of $\mathrm{X} /$ gamma ray sources which usually display signatures of different components in emission and absorption. In July 2012 we observed with X-shooter a small sample of counterparts of bright IGR sources in order to better determine their physical properties. We present the first results of these observations.
\end{abstract}

'An INTEGRAL view of the high-energy sky (the first 10 years)' 9th INTEGRAL Workshop and celebration of the 10th anniversary of the launch,

October 15-19, 2012

Bibliotheque Nationale de France, Paris, France

\footnotetext{
${ }^{*}$ Speaker.

$\dagger$ Based on observations collected at the European Organization for Astronomical Research in the Southern Emisphere, Chile under Program P089.D-0056(A)
} 


\section{Introduction}

INTEGRAL has discovered several bright sources in the Galactic plane, they are usually divided in two groups: obscured sources and SFXTs [2]. Their study is difficult due to their distance (>1-15 kpc), absorption (line-of-sight and intrinsic) and the crowded fields in which they are found. Since their first discovery, several of them have been identified as High Mass X-ray Binaries. The vast majority of them are quite bright in infrared and faint in the visible range due to extremely high absorption ( $\mathrm{A}(\mathrm{V})$ almost always greater than 10). Therefore the spectroscopic observations have almost always been performed in infrared and for some of these sources, no visible spectrum is available. This is a problem because the best diagnostics of the properties of the primary star such as rotation, metallicity and mass loss lie in the visible and ultraviolet domains (see e.g. [8] ). For the sources that do have visible spectra, they are not simultaneous with the infrared ones which may make the comparison unreliable due to the intrinsic variability of the primary star at least concerning the emission features.

The X-shooter spectrograph [17] with its exceptional wavelength range and its high sensitivity is very well placed to investigate on these matters allowing to monitor the known NIR spectrum while exploring the VIS and UVB spectrum whenever possible. In principle the comparison of the simultaneous visible and infrared diagnostics should allow to constrain strongly the properties of the primary star. Moreover the line of sight absorption can be studied thoroughly by measuring the total reddening with the SED and measuring the equivalent width and, for identified lines, the Doppler shifts of ISM absorption features. We asked for 2.5 nights of French GTO time to observe a sample of eleven IGR sources in order to perform a first test of X-shooter capabilities on these sources. We aimed at observing all the sources of the sample at least twice to check for variable features. Unfortunately, due to bad weather, we could observe only one night and therefore only seven sources could be observed once. Here we report on the first results of these observations for three of the sources in our sample.

\section{Observations and Data Reduction}

The main observations consisted of four different pointings of $300 \mathrm{~s}$ each taken using the nodding along the slit technique with an offset of 5 arcsec between pointings in a standard ABBA sequence. In order to avoid saturation, all the exposures on the NIR arm and some of those in the VIS arm were split in shorter integrations. All of these pointings were performed using narrow slits ( $0.5^{\prime \prime}$ for the UVB arm, $0.7^{\prime \prime}$ for the VIS arm and $0.6^{\prime \prime}$ for the NIR arm) (see table 1 for details). Each source was also briefly observed with a wide (5.0") slit to estimate the slit losses. A telluric A0V star was observed before each source and a flux standard was observed in the beginning of the night.

The spectra were reduced using the X-shooter pipeline [6, 9]. We used the pipeline to build calibration files (master bias, wavelength solution...) from daytime calibration observations. Afterwards we performed bias and dark subtraction followed by division by a master flat field. The orders were then extracted, rectified in wavelength-slit space and then shifted and added to subtract the sky contribution. The resulting $2 \mathrm{D}$ order spectra were then merged to produce the final $2 \mathrm{D}$ spectrum and the 1D spectrum was extracted using a PSF-weighing scheme in IDL. The 1D spectra 
were subsequently normalized. Flux calibrations and telluric corrections have not been performed yet.

Table 1: Summary of the narrow slit observations for the three sources presented here. Four independent pointings of 300s each were performed in a standard nodding ABBA sequence. Each pointing in the NIR arm was split in 20 independent exposures each one with up to 6 independent integrations that are averaged by the detector. The exposure time is given as $\mathrm{N}$ (exposure) x $\mathrm{N}$ (integrations) $\mathrm{x}$ (Detector Integration Time or DIT).

\begin{tabular}{cccc}
\hline Source Name & $\begin{array}{c}\text { Coordinates } \\
(\text { J2000) }\end{array}$ & $\begin{array}{c}\text { Exptime (s) } \\
\text { UVB, VIS, NIR }\end{array}$ & $\begin{array}{c}\text { Slit Width (') } \\
\text { UVB, VIS, NIR }\end{array}$ \\
\hline IGR J16465-4507 & $164635-450704$ & $4 \times 300,4 \times(2 \times 150), 20 \times(2 \times 30)$ & $0.5^{\prime \prime}, 0.7^{\prime \prime}, 0.6^{\prime \prime}$ \\
\hline IGR J18483-0311 & $184817-031015$ & $4 \times 300,4 \times 300,20 \times(6 \times 10)$ & $0.5^{\prime \prime}, 0.7^{\prime \prime}, 0.6^{\prime \prime}$ \\
\hline IGR J16418-4532 & $164151-453225$ & $4 \times 300,4 \times 300,20 \times(2 \times 30)$ & $0.5^{\prime \prime}, 0.7^{\prime \prime}, 0.6^{\prime \prime}$ \\
\hline
\end{tabular}

\section{IGR J16465-4507}

This source has been classified as a supergiant of spectral type B0-B1 ([1], [2]) or O9.5 [13]. Spectroscopic observations have been performed from the U (3900 $⿱$ ) to the K band. We clearly detect IGR J16465-4507 in the UVB arm starting from about $3500 \AA$. The UVB spectrum looks similar to the one shown by [11. The possible presence of the HeII 4686 line $\AA$ suggests a B0.5B1 spectral type [⿰]月. The width of thin lines such as e.g. SiIII $4552 \AA$ suggests fast rotation with vsini greater than $200 \mathrm{~km} / \mathrm{s}$. The high rotation velocity implies that we're observing the source at high inclination. The VIS spectrum is also quite bright showing an intense $\mathrm{H} \alpha$ feature (not shown) and several He I features.
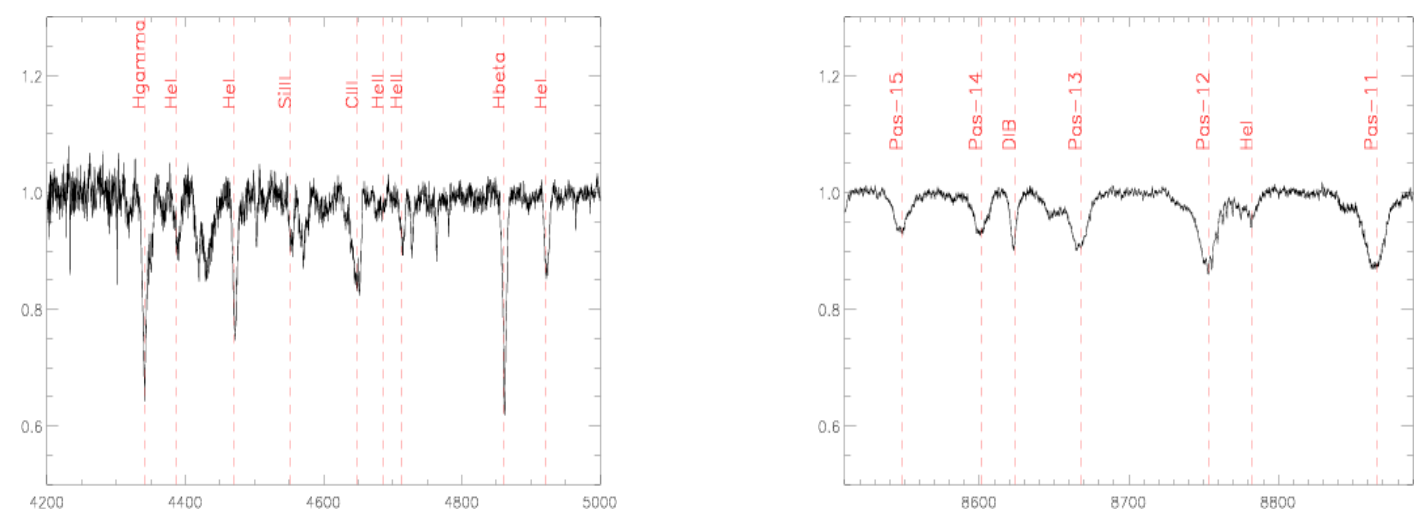

Figure 1: Left: Section of the UVB spectrum of IGR J16465-4507, the possible presence of a weak HeII $4686 \AA$ Aine suggests a spectral type B0.5-B1 [11] as for an O star this line would be stronger. Right: Section of the I band spectrum of IGR J16465-4507, containing several Paschen lines and the 8620 A DIB with equivalent width $\sim 0.5 \AA$.

In figure 1 we show the first I band spectrum of IGR J16465-4507, preliminary comparisons with the I band spectra of Westerlund 1 supergiant stars [12] also suggest a B0.5-B1 spectral type. 

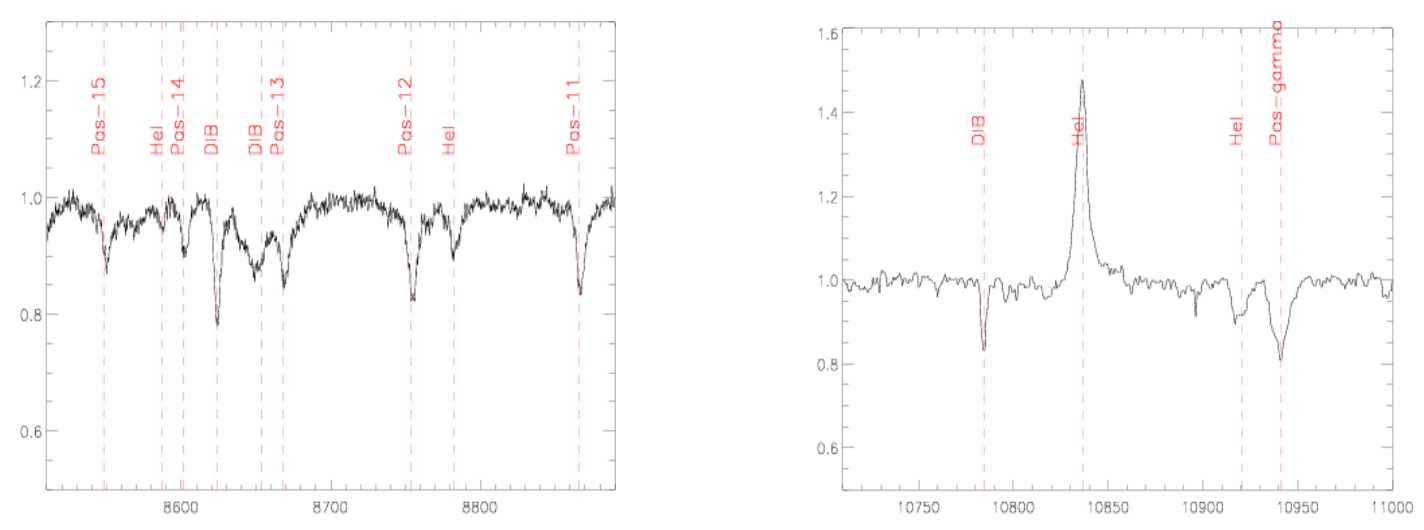

Figure 2: Left: section of the I band spectrum of IGR J18483-0311 containing several Paschen lines and the DIBs at $8620 \AA$ and $8643 \AA$. The equivalent width of the $8620 \AA$ line is $\sim 1.2 \AA$. Right: Section of the $\mathrm{J}$ band spectrum of IGR J18483-0311 containing the HeI $10830 \AA$ Aline in emission, the HeI $10920 \AA$ and Brackett $\gamma$ lines in absorption. The HeI $10830 \AA$ emission line has a slightly asymmetric profile which may be of P Cygni type however a nore careful analysis is required. The feature at $10780 \AA$ proposed as a possible DIB by [T] is also present.

More quantitatively, we estimated the spectral type with the properties of the Paschen lines using the approach of [1]. We measured the equivalent width of the Paschen-11 and Paschen-17 lines obtaining $\sim 2.4$ and $\sim 0.6 \AA$ respectively. These values, once compared to Figure 7 and 8 (last panel) of [四] again point toward a spectral type B0.5- B1 with Teff $\sim 25000 \mathrm{~K}$ and $\operatorname{logg} \sim 3$.

We also remark that the $8620 \AA$ DIB is clearly detected with $\mathrm{EW} \sim 0.5 \AA$. This is expected considering the source absorption. The equivalent width of this DIB has been proposed as a reddening indicator by [10]. Using their formula we can estimate $\mathrm{E}(\mathrm{B}-\mathrm{V}) \sim 1.36$ which implies $\mathrm{A}(\mathrm{V})$ $\sim 4.2$. This value is outside by about one magnitude of the $90 \%$ confidence range estimated by [14], however using $R(V)=4$, we obtain $A(V)=5.44$, well inside their confidence range. This may suggest a non standard extinction law in this direction.

\section{IGR J18483-0311}

IGR J18483-0311 has been classified as a B0.5 supergiant with NIR spectroscopic observations [15] and it has also been observed non simultaneously in optical [5], [16]. We detected IGR J18483-0311 only starting from about $6000 \AA$, a weak $\mathrm{H} \alpha$ line is visible, similar to [5]. From the I band spectrum in Figure 2 we measured the equivalent width of the Paschen-11 and Paschen-17 lines obtaining respectively $2.1 \AA$ and $0.6 \AA$ thus validating the spectral classification by [15]. We detect the $8620 \AA \mathrm{DIB}$ with $\mathrm{EW} \sim 1.2 \AA$ and therefore $\mathrm{E}(\mathrm{B}-\mathrm{V}) \sim 3.26$ which is in good agreement with published photometry [15]. From it we obtain $A(V)=10.2$ for $R(V)=3.1$, smaller than 15.7 as in [15]. Again a non standard extinction law with $R(V) \sim 4.8$ could explain this discrepancy.

In the NIR spectra we identify for the first time in this source a bright, slightly asymmetric $\mathrm{HeI}$ $10830 \AA$ emission line (see Figure 2). This line was not reported in the NIR spectrum of [15], this detection may imply variability in the wind of the source. The asymmetry we detect may indicate the presence of a P Cygni profile. 

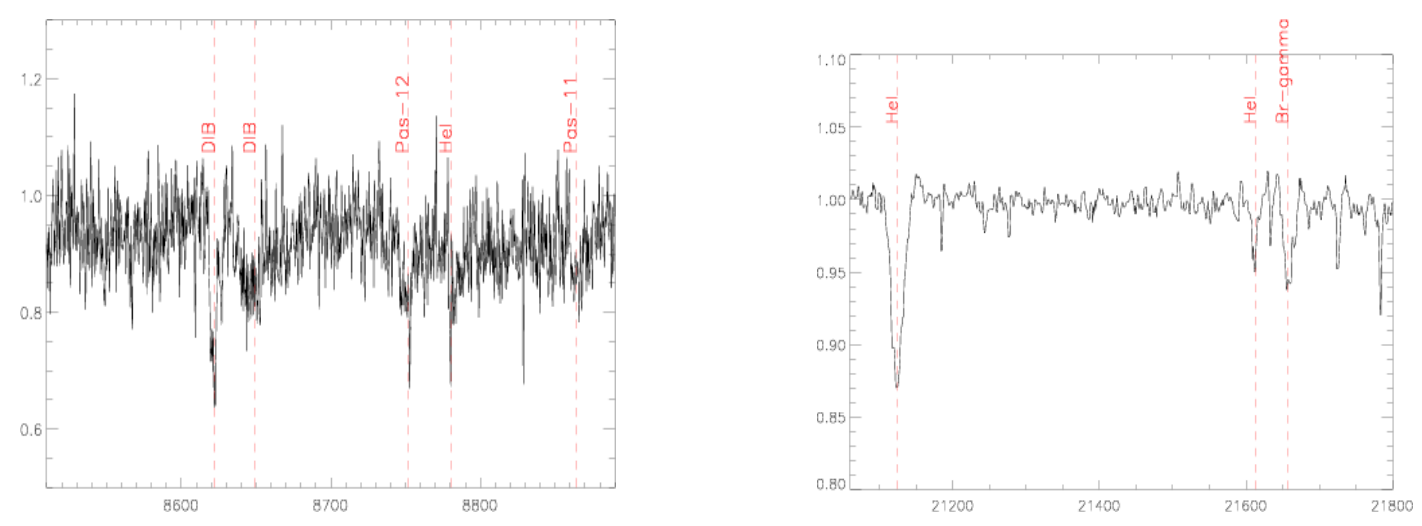

Figure 3: Left: Section of the I band spectrum of IGR J16418-4532. The low S/N allows only the detection of the strong Paschen lines and of diffuse interstellar bands. Right: Section of the $\mathrm{K}$ band spectrum of IGR J16418-4532 containing HeI $21126 \AA$, He I $21614 \AA$ and Brackett $\gamma$ mainly in absorption. They also show small emission bumps consistent with the presence of moderate winds. The absorption feature not marked between He I $21614 \AA$ and Brackett $\gamma$ is a telluric feature.

\section{IGR J16418-4532}

IGR J16418-4532 has been classified as an 08.5-O9.5 supergiant [2] on the basis of photometric observations and SED fitting but no spectroscopic observations have been reported yet. The source has been detected with a low S/N (<10) in the VIS arm (Fig. 3). The Paschen lines 11 and 12 are visible and the continuum is quite faint. We therefore cannot use this part of the spectrum for a spectral type classification. The $8620 \AA$ DIB absorption has equivalent width $\sim 1.65 \AA$ implying $\mathrm{E}(\mathrm{B}-\mathrm{V}) \sim 4.49$ and $\mathrm{A}(\mathrm{V})=13.9$ for $\mathrm{R}(\mathrm{V})=3.1$ consistent with [14]. Conversely the NIR spectrum has a good $\mathrm{S} / \mathrm{N}$ ratio but it does not present strong emission lines. We therefore try to establish a classification using absorption features in the NIR spectrum. In the $\mathrm{K}$ band two common features for $\mathrm{O}$ and $\mathrm{B}$ supergiants are the HeI $21126 \AA$ and the Brackett $\gamma$. Their equivalent widths are respectively $\sim 2.8 \AA$ and $\sim 1.2 \AA$. According to $[\rrbracket$, these EWs, especially the high value for HeI $21126 \AA$, point towards an 09.5 spectral type (see their figure 24 ). These lines also display small emission excesses which may be due to wind emission (Fig. 3).

\section{Conclusions}

We have presented the first results of our X-shooter observations of a sample of IGR sources. Taking advantage of the unprecedented spectral coverage, we could compare the results in different spectral domains. On this basis we suggest that IGR J16465-4507 is a B0.5-B1 supergiant star in fast rotation, probably seen at high inclination. We confirm the classification of IGR J18483-0311 as a B0.5 supergiant and we detect for the first time a bright He I emission line possibly suggesting enhanced mass loss with respect to previous observations. We present the first VIS and NIR spectra of IGR J16418-4532 and on the basis of the K band spectra we suggest that the star is a 09.5 supergiant.

Using the detection of the $8620 \AA$ DIB in the three spectra we estimate the reddening in the direction of these sources. The results are compatible with published photometry when available. 
However in two of three cases the extinction estimations we obtain are compatible with published ones only if we assume non standard extinction curves.

\section{References}

[1] Caron, G. et al., 2003, ”The lack of Blue Supergiants in NGC 7419, a Red Supergiant-Rich Galactic Open Cluster with rapidly Rotating Stars", ApJ 126, 1415

[2] Chaty, S.. et al., 2008, "Multi-wavelength observations of Galactic hard X-ray sources discovered by INTEGRAL. I. The nature of the companion star", A\&A 484, 783

[3] Evans et al., 2004, ”A 2dF survey of the Small Magellanic Cloud”, MNRAS 353, 601

[4] Hanson, M.M. et al. 1996, ”A Spectral Atlas of Hot, Luminous Stars at 2 Microns”, ApJS, 107, 281

[5] Masetti, N. et al., 2008, "Unveiling the nature of INTEGRAL objects through optical spectroscopy. VI. A multi-observatory identification campaign", A\&A 482, 113

[6] Goldoni, P. et al., 2006, ”Data reduction software of the X-shooter spectrograph”, Proc. SPIE 6269, 80

[7] Groh, J.H., et al., 2007, ”Spectral atlas of massive stars around He I 10830 ^”, A\&A 465, 993

[8] Martins, F., 2010, " UV, optical and near-IR diagnostics of massive stars", Proceedings of the 39th Liege Astrophysical Colloquium, ArXiv:1010.5347

[9] Modigliani, A., et al. 2010, ”The X-shooter pipeline”, Proc. SPIE 7737, 56

[10] Munari, U. et al. 2008, ” Diffuse interstellar bands in RAVE survey spectra”, A\&A 488, 969

[11] Negueruela, I. et al., 2006, ”Supergiant Fast X-ray Transients: A New Class of High Mass X-ray Binaries Unveiled by INTEGRAL", Proc. ESA-SP "The X-ray Universe", 165

[12] Neguereula, I. et al. 2010, "The population of OB supergiants in the starburst cluster Westerlund 1", A\&A 516, 78

[13] Nespoli, E. et al., 2008, ”Unveiling the nature of six HMXBs through NIR spectroscopy”, A\&A 486, 911

[14] Rahoui, F. et al., 2008, "Multi-wavelength observations of Galactic hard X-ray sources discovered by INTEGRAL, II. The environment of the companion star” , A\&A, 484, 801

[15] Rahoui, F. \& Chaty, S., 2008, ”IGR J18483-0311: a new intermediate supergiant fast X-ray transient”, A\&A 492, 363

[16] Torrejon, J.M. et al., 2010, ”Near-infrared survey of high mass X-ray binary candidates”, A\&A 510, 61

[17] Vernet, J. et al., 2011, "X-shooter, the new wide band intermediate resolution spectrograph at the ESO Very Large Telescope”, A\&A 536, A105 